\title{
Identification and functional characterization of novel GDAP1 variants in Chinese patients with Charcot-Marie-Tooth disease
}

\author{
Zhi-Ying $\mathrm{Wu}^{1}$, Cong-Xin Chen ${ }^{2}$, Jia-Qi $\mathrm{Li}^{3}$, Hai-Lin Dong ${ }^{3}$, and Ge Bai ${ }^{4}$ \\ ${ }^{1}$ Second Affiliated Hospital,Zhejiang University School of Medicine \\ ${ }^{2}$ First Affiliated Hospital of Fujian Medical University \\ ${ }^{3}$ 1. Zhejiang University School of Medicine Second Affiliated Hospital, Department of \\ Neurology Hangzhou, Zhejiang, CN \\ ${ }^{4} \mathrm{NHC}$ and CAMS Key Laboratory of Medical Neurobiology, MOE Frontier Science Center \\ for Brain Research and Brain-Machine Integration, School of Brain Science and Brain \\ Medicine, Zhejiang University
}

June 2, 2020

\begin{abstract}
Variants in the ganglioside-induced differentiation associated protein 1 (GDAP1) gene is responsible for a demyelinating form of Charcot- Marie-Tooth disease (CMT4A), an axonal form of CMT2K and an intermediate form of CMTRIA. In this study, multiplex ligation-dependent probe amplification (MLPA) and whole-exome sequencing (WES) were performed in 30 unrelated CMT patients. We identified 10 pathogenic variants in 3 known CMT related genes, including 3 novel variants (p.L26R, p.S169fs, c.694+1G>A) and one known pathogenic variant (p.R120W) in GDAP1. Functional studies were performed and the pathogenicity of novel GDAP1 variants were classified. The effect of c.694+1G>A on pre-mRNA was verified via minigene splice assay. Cellular biological effects showed an ultrastructure damage of mitochondrial, reduced protein levels, different patterns of mitochondrial dynamics, decreased mitochondrial membrane potential $(\Delta \psi \mathrm{m})$, ATP content, and defects in respiratory capacity in the patient carrying p.L26R and p.S169fs in GDAP1. Further we described the clinical features of patients carrying pathogenic variants in GDAP1 and found that almost all Chinese CMT patients with GDAP1 variants present axonal type. Our results broaden the genetic spectrum of GDAP1 and provided functional evidence for mitochondrial pathways in the pathogenesis of GDAP1 variants.
\end{abstract}

\section{Introduction}

Charcot-Marie-Tooth disease (CMT) caused by pathogenic variants in the ganglioside-induced differentiation associated protein $1(G D A P 1)$ gene is clinically characterized by a severe distal motor and sensory neuropathy. But a broad spectrum of phenotypes should be considered because of the variability in different inheritance patterns (Azzedine et al., 2003; Manganelli et al., 2012). Phenotype-genotype correlations have been rarely investigated in Asian area besides a few cases reported (Abe et al., 2011; Chung et al., 2011; Fu et al., 2017; Lin et al., 2011; Pakhrin et al., 2018; Zhang et al., 2004). The locus of chromosome 8q13.q21 was first identified to be linked with recessive CMT cases in Tunisian families (Ben Othmane et al., 1993), then the corresponding gene, GDAP1, was found in the previous study (Baxter et al, 2002). Unlike many other CMT disease-causing genes, it is associated with an axonal (CMT2K; MIM\# 607831), a demyelinating (CMT4A; MIM\# 214400) or an intermediate form (CMTRIA; MIM\# 608340) of CMT with recessive or dominant modes of inheritance (Cassereau et al., 2011).

GDAP1 gene encodes a 358-amino acid protein. As an integral membrane protein of the external mitochondrial membrane, it is expressed ubiquitously in various tissues but mainly expressed in neurons (Niemann, 
Ruegg, La Padula, Schenone, \& Suter, 2005; Pedrola et al., 2008). However, the explicit molecular mechanism underlying GDAP1 function remains unclear. Available data indicate an important role of GDAP1 in many aspects of mitochondrial morphology and functioning such as mitochondrial dynamics (Lopez Del Amo et al., 2015; Niemann et al., 2005; Niemann, Wagner, Ruegg, \& Suter, 2009; Pedrola et al., 2008), formation and/or modulation of the ER-mitochondria contacts (Pla-Martin et al., 2013) and mitochondrial transport (Estela, Pla-Martin, Sanchez-Piris, Sesaki, \& Palau, 2011; Pla-Martin et al., 2013). A recent study showed that neuroinflammation was also involved in the pathophysiology of axonal GDAP1 -related CMT (Fernandez-Lizarbe et al., 2019).

Herein, we provide the clinical features, frequency of distribution and physiopathology of patients with novel GDAP1 variants in a cohort of southeast Chinese patients with CMT by performing multiplex ligationdependent probe amplification (MLPA) and whole-exome sequencing (WES). Then the variants were further characterized by functional studies.

\section{Materials and methods}

\section{Subjects}

Thirty unrelated patients clinically diagnosed with CMT were enrolled consecutively between September 2018 and August 2019 from southeastern China. Clinical evaluations and neurological examinations were performed by at least two senior neurologists. Five hundred unrelated phenotypically normal controls with no known family history of CMT were included in the study. Written informed consent was obtained from all the subjects or their guardians. This study was approved by the ethics board of Second Affiliated Hospital, Zhejiang University School of Medicine.

\section{DNA extraction andwhole exome sequencing}

Genomic DNA samples were extracted from peripheral blood lymphocytes using a DNA isolation kit (Qiagen, Germany). WES was performed using the Agilent SureSelect Human All Exome V6 kit (Agilent Technologies Inc, Canada) on an Illumina Hiseq X Analyzer (Illumina, USA) with 150-bp pairedend runs. SIFT (http://sift.jcvi.org/), PolyPhen-2 (http://genetics. bwh.harvard.edu/pph2/), and MutationTaster (http://www.mutationtaster.org/) were used to predict the possible protein functional change caused by a variant. The frequency in the general population of the identified variants was checked using the Single Nucleotide Polymorphism (dbSNP) Database (https://www. ncbi.nlm.nih.gov/snp/), Exome Aggregation Consortium (ExAC) database (http://exac.broadinstitute.org/), the 1000 Genomes Project (https://www.ncbi.nlm.nih.gov/variation/tools/1000 genomes/) and The Genome Aggregation Database (http://gnomad-old.broadinstitute.org/). All the potential variants were validated by Sanger sequencing.

\section{Minigene assay}

Exonic and at least $150 \mathrm{bp}$ of flanking 5' and 3' intronic GDAP1 sequences (RefSeq NM_018972) from genomic DNA of proband of family 2 and control were PCR-amplified using KOD-Plus-Neo DNA Polymerase (TOYOBO, Japan), with primers carrying restriction sites for XhoI and EcoRI (5'-GGTACGGGATC ACCAGAATTCTCCACTCGCCGTTTAACTC-3' (forward) and 5'-ATCCTGCAG CGGCCGCTCGAGCCCACGTGCTGGTCCTTATT-3' (reverse). PCR products were subcloned into the splicing reporter pSPL3 vector using T4 DNA Ligase (New England Biolabs, USA). The pSPL3 vector contains a minigene. Transcription occurs from the SV40 promoter and the RNA undergoes splicing under control of the host cell's RNA splicing machinery, resulting in the fusion of two vector exon sequences. If a genomic DNA fragment cloned into pSPL3 contains an exon with functional splice donor (SD) and splice acceptor (SA) sequences, a different splicing pattern (the complete skipping of genomic DNA fragment) may occur. Minigene constructions were confirmed by Sanger sequencing. For minigene assay, HEK293T cells were maintained at $37^{\circ} \mathrm{C} / 5 \% \mathrm{CO}_{2}$ in Dulbecco's modified Eagle's medium (DMEM) (GIBCO, USA) supplemented with $10 \%$ fetal bovine serum (FBS) (GIBCO, USA), $100 \mathrm{IU} / \mathrm{ml}$ penicillin and $100 \mu \mathrm{g} / \mathrm{ml}$ streptomycin (HyClone, USA). Cells were transfected with expression vectors using Lipofectamine 3000 (Invitrogen, USA) according to the manufacturer's instructions. After 36 hours, total RNA was extracted by 
using RNAiso Plus (Takara, Japan) and then was reverse transcribed into cDNA using the PrimeScript RT reagent Kit (Takara, Japan), following the manufacturer's protocol. Finally, PCR products were visualized in $10 \%$ agarose gels to study the transcript band pattern and then identify potential changes in splicing process by Sanger sequencing.

\section{Primary fibroblasts culture and treatment}

Primary fibroblast cell lines were established from skin biopsies with punches (Electron microscopy sciences, USA), acquired from the proband of family 2. Written consent was obtained from the guardians of this patient. Fibroblasts were maintained in DMEM (GIBCO, USA) with low glucose $(1 \mathrm{~g} / \mathrm{L})$ and sodium pyruvate supplemented with $10 \%$ FBS (GIBCO, USA).

\section{Transmission Electron microscopy}

Muscular biopsy was performed in the proband of family 2 to observe ultrastructure of mitochondria in muscular tissue. Muscular tissue was fixed in 2.5\% glutaraldehyde (Sangon Biotech, China) in PBS at $4^{\circ} \mathrm{C}$ for $2 \mathrm{~h}$. Then, the tissue block was washed three times with PBS, and the block embedded with agar were fixed again with $1 \%$ osmium tetroxide for $2 \mathrm{~h}$ then washed three times with PBS. The tissue block was dehydrated with an ethanol series and fixed again with Epon 812 (Sangon Biotech, China). The ultrathin (70 $\mathrm{nm}$ ) sections were collected on Formvar/carbon-coated nickel grids (Sangon Biotech, China), and the grids were stained with 2.5\% uranyl acetate (Sangon Biotech, China) for 7 min followed by lead citrate (Sangon Biotech, China) for $2.5 \mathrm{~min}$, and then the sections were observed with a JEM-1011 electron microscope (JEOL, Japan).

\section{Western blotting}

The cells were harvested in the cell lysis reagent (Sigma, USA) supplemented with a $1 \%$ protease inhibitor PMSF (Beyotime, China) and centrifuged at 13,000 rpm for 20 min at $4{ }^{\circ} \mathrm{C}$. Western blot was performed as previously described ( $\mathrm{Li}, \mathrm{Liu}, \mathrm{Liu}, \mathrm{Lu}, \& \mathrm{Wu}, 2017$ ). Protein extracts were separated electrophoretically on 10\% SDS-PAGE gels and immunoblotted with primary antibodies (Abnova, Taiwan, China). HRP-conjugated secondary antibodies (Santa Cruz, USA) were used to detect primary antibodies. The densitometric values of the bands of three independent experiments were analyzed using Adobe Photoshop software and were normalized to $\beta$-Tubulin levels.

\section{Confocal microscopy}

Primary fibroblasts growing in 35-mm glass bottom dishes (Sangon Biotech, China). Cells were incubated with MitoTracker@ Red (Invitrogen, USA) and DAPI Reagent (Sigma, USA) and then were directly observed under a confocal microscope (Leica, Germany).

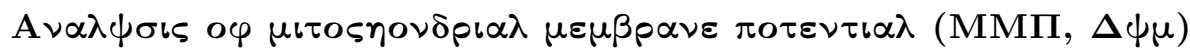

Mitochondrial membrane potential was determined using tetramethylrhodamine methyl ester perchlorate, TMRM (Invitrogen, USA). Cells were fixed and stained according to the manufacture's recommended protocol. An Olympus microscope equipped with a CCD camera was used for acquisition of fluorescent images. The average fluorescent intensities were quantified using ImagePro Plus version 5.0 imaging software.

\section{Measurement of ATP content}

ATP content was determined in fibroblasts lysates using an ATP bioluminescence assay kit (Perkin Elmer, USA), according to the manufacture's recommended protocol. Measurements were performed in a luminometer (Perkin Elmer, USA).

\section{Measurement of oxygen consumption}

The rate of oxygen consumption in fibroblasts was measured with a Seahorse Bioscience XF-96 extracellular flux analyzer (Seahorse Bioscience, USA). XF96 creates a transient $7 \mu l$-chamber in specialized microplates that allows for the determination of oxygen and proton concentrations in real time. To allow comparison 
between different experiments, data are expressed as the rate of oxygen consumption in $\mathrm{pmol} / \mathrm{min}$ or the rate of extracellular acidification in $\mathrm{mpH} / \mathrm{min}$, normalized to cell protein in individual wells determined by the Bradford protein assay (Bio-Rad, USA). A density of 20000 cells per well in 96-well plate was coated with Cell-Tak ${ }^{\mathrm{TM}}$ adhesive. The rate of $\mathrm{O} 2$ was determined under basal condition and the addition of oligomycin $(1.5 \mu \mathrm{M})$, carbonyl cyanide $p$-(trifluoromethoxy) phenylhydrazone $(\mathrm{FCCP})(0.5 \mu \mathrm{M})$, rotenone $(1 \mu \mathrm{M})$ and antimycin A $(1 \mu \mathrm{M})$.

\section{Statistical analysis}

All the values are expressed as the mean $\pm \mathrm{SD}$, and the data were analyzed by one-way ANOVA or two-way ANOVA using GraphPad Prism software. P values less than 0.05 were considered statistically significant.

\section{Results}

\section{Genetic analysis}

MLPA and WES were performed on 30 unrelated CMT patients. Eight patients had PMP22duplication. Ten variants in 3 known genes related to CMT, including 3 novel variants (p.L26R, p.S169fs, c.694+1G>A) and one known variant (p.R120W) in GDAP1 gene (Fig. 1A, B ) and 6 known pathogenic variants of other genes (p.R280H and p.R259L in MFN2 ; p.V181M, c.-103C>T, p.V91M and p.R183H in GJB1) (Supp. Fig. S1 ), were identified in 9 index patients. No variant was detected in the remaining 13 cases. The 3 novel variants were absent or with low frequency in several global human genome databases such as 1000G and ExAC. After assessing by SIFT, PolyPhen-2 software and MutationTaster, all of them were predicted to cause deleterious disruptions to the protein. According to American College of Medical Genetics and Genomics (ACMG) standards and guidelines, 2 were assigned as 'pathogenic variants' and 1 as 'variants with uncertain significance'(Table 1) .

Besides the three families carrying variants in GDAP1 in this study, we have previously reported three GDAP1 variants (p.W31X, p.Q38X, p.V215I) in 2 patients (Chen et al., 2019). The location of 7 variants from our studies and 10 variants from other Chinese studies in the structure of GDAP1 protein are shown in Fig. 1C . Taken all the probands in our studies into consideration, we can summarize a frequency of $2.78 \%$ (5/180) in GDAP1 in our CMT patients, which is similar to those reported in other Chinese studies (Fu et al., 2017; Pakhrin et al., 2018).

\section{Splicing assays of novel variantc.694 $+1 \mathrm{G}>\mathrm{A}$ in $G D A P 1$}

To validate the functional classification of the novel splicing variant c.694+1G $>$ A identified in family 1 , minigene splice assay was conducted. Exon 5 (115bp) and 150bp of flanking 5' and 3' intronic sequences in GDAP1 gene from genomic DNA of proband of family 1 and control were subcloned into the pSPL3 vector. Mutant and control minigene were transfected into HEK293 T cells. RNA was extracted and reversetranscribed into cDNA after plasmid transfection of cells for $48 \mathrm{~h}$. Agarose gel electrophoresis showed a mutant band that is smaller than the WT band (Fig. 2A). Sequencing analysis showed a loss of Exon 5 (115bp) in mutant minigene (Fig. 2B) . The schematic diagram of the affected splicing products is shown inFig. 2C . These results verified the effect of the c.694+1G $>$ A in GDAP1 on pre-mRNA.

\section{Functional analyses of novel variants p.L26R and p.S169fs in GDAP1}

To elucidate the biological effects of the novel variants p.L26R and p.S169fs identified in family 2, we studied their function in muscle tissue and fibroblasts from the proband of family 2. Transmission electron microscopy was used to observe the ultrastructure in muscle tissue of patient. In Fig. $\mathbf{3}$, obvious damage of mitochondrial structure including disorganization of cristae and disruption of the mitochondrial membrane were found in muscle tissue of the patient carrying p.L26R and p.S169fs variants. We further analyzed the expression level of mutant GDAP1. Western blot results revealed that mutant protein in muscle tissue of the patient was markedly reduced compared to that of WT GDAP1 (Fig. 4A, B ). Actually, mutant protein in patient-derived fibroblasts also showed an undetectable band compared to that of WTGDAP1 (Supp. Fig. S2A, B ). 
To evaluate the mitochondrial distribution pattern, mitochondria were visualized using Mitotraker Red staining. We found the mitochondrial network was more fragmented and contained a larger number of short mitochondria in patient-derived fibroblasts than control fibroblasts (Fig. 4C ). Additionally, tetramethylrhodamine methyl ester perchlorate (TMRM) was used to validate the level of mitochondrial membrane potential (MMP, $\Delta \psi \mathrm{m}) . \Delta \psi \mathrm{m}$ is shown in red represented by the relative intensity of the TMRM signal. We found the optical intensity was reduced by $26.2 \%$ in patient-derived fibroblasts compared with control 1 $(\mathrm{n}=3, \mathrm{p}=0.0048)$, and $23.9 \%$ compared with control $2(\mathrm{n}=3, \mathrm{p}=0.0035)$ (Fig. 4D, $\mathbf{E})$. To further determine whether intracellular reactive oxygen species (ROS) showed increased production due to the loss of $\Delta \psi \mathrm{m}$, MitoSOX Red, a fluorogenic dye for selective detection of superoxide in the mitochondria of live cells was used. The result showed that no significant changes of ROS level between patient-derived fibroblasts and control fibroblasts (Supp. Fig. S2C ).

The capacity of oxidative phosphorylation in mutant and control fibroblasts was examined by measuring the levels of cellular and mitochondrial ATP using a luciferin/luciferase assay. Fibroblasts were incubated in the media in the presence of glucose, and 2-deoxy-d-glucose with pyruvate. As shown in Fig. $\mathbf{4 F}$, the levels of ATP production in mutant fibroblast in the presence of glucose (total cellular levels of ATP) decreased by $44.6 \%$ compared with those measured in the control fibroblast $(\mathrm{n}=5, \mathrm{p}<0.0001)$. By contrast, as shown inFig. 4G , the levels of ATP production in mutant fibroblasts in the presence of pyruvate and 2-deoxy-dglucose which can inhibit the glycolysis (mitochondrial levels of ATP) decreased by $40.3 \%$ relative to control fibroblasts $(\mathrm{n}=5, \mathrm{p}<0.0001)$. ATP bioluminometers analysis revealed that the relative ATP production was apparently decreased in the patient-derived fibroblasts.

To further evaluate alterant cellular bioenergetics in mutant fibroblasts, we examined the oxygen consumption rates (OCR) of it and control fibroblasts. As shown in Fig. $\mathbf{4 H}$ andFig. $\mathbf{4 I}$, the basal OCR in the mutant cell lines decreased by $13.2 \%(\mathrm{n}=3, \mathrm{p}=0.0056)$ relative to the control fibroblasts. To investigate which of the enzyme complexes of the respiratory chain was affected in the mutant cell lines, OCR were measured after the sequential addition of oligomycin (to inhibit the ATP synthase), FCCP (to uncouple the mitochondrial inner membrane and allow for maximum electron flux through the ETC), rotenone (to inhibit complex I) and antimycin A (to inhibit complex III). The ATP-linked OCR, maximal OCR and reserve capacity in mutant fibroblasts decreased by $16.5 \%, 15.1 \%, 15.3 \%(\mathrm{n}=3, \mathrm{p}=0.0113,0.0114,0.0335)$ relative to the control fibroblasts, respectively. However, proton leak OCR and non-mitochondrial OCR showed no significance between mutant and control fibroblasts.

\section{Clinical features of patients with GDAP1 variants}

Three novel and one known pathogenic variants in GDAP1 gene were identified in three CMT families (Fig. 1A ), which showed axonal type of neuropathy. In family 1, the proband with a homozygous splicing variant c. $694+1 \mathrm{G}>\mathrm{A}$ was a 30 -year-old man without family history of consanguinity. Onset of the disease was at age 5 . He had difficulty in walking and hand manipulation and received 3 times surgery of deformity correction due to the severe foot deformity. The patient complained about obvious weakness and muscle atrophy in all the limbs. Additionally, he had sensory loss and depressed deep tendon reflexes. The other affected individuals in this family include the proband's younger brother and cousin. They carried the same variant and also had foot deformities and progressive distal muscle weakness and atrophy in all the limbs.

In family 2, the proband carrying p.L26R and p.S169fs was a 5-year-old boy with a history of unsteady gait and foot drop for a year. The disease presented slowly progressive. Symptoms of his foot drop began from right to left. The distal muscle atrophy began in his feet, followed by leg muscle involvement. He had a severe foot deformity and received a surgery of deformity correction at age of 5 . Neurological examinations showed distal muscle weakness and atrophy in lower limbs, without impairment of proximal muscles. All deep-tendon reflexes were weakened.

The proband of family 3 harbored a known missense mutations p.R120W. She was a 73-year-old female with a history of distal weakness and sensory loss in the lower limbs for over 10 years. Gradually, she had difficulty in walking and climbing stairs. She could get around only using a crutch since the age of 72 . No 
family history was identified. The detailed clinical features of patients carrying GDAP1 pathogenic variants were shown in Table 2 .

\section{Discussion}

In this study, we applied MLPA and WES in 30 unrelated clinically diagnosed CMT patients from southeast China. Finally, 17 out of 30 patients were genetically diagnosed with CMT, including 8 patients withPMP22 duplication, 4 with known GJB1 variants, 3 with GDAP1 variants (3 novel and 1 known) and 2 with known MFN2variants. Herein, we focus on the three novel variants (p.L26R, p.S169fs, c.694+1G >A) and one known variant (p.R120W) in GDAP1. Taking 3 variants previously reported in 2 patients into consideration (Chen et al., 2019), we can summarize a frequency distribution of $2.78 \%(5 / 180)$ in GDAP1 . Previous studies in the Chinese population showed the similar frequency (Fu et al., 2017; Pakhrin et al., 2018). In studies conducted in other Asian CMT patients, the frequency ranged from 1 to 2.8\% (Abe et al., 2011; Lin et al., 2011; Yoshimura et al., 2017), which was generally lower than those reported in the Mediterranean populations (5.4\%-11.5\%) (Claramunt et al., 2005; Manganelli et al., 2014; Pezzini et al., 2016; Sivera et al., 2013; Zimon et al., 2015).

It is noticeable, however, all the patients with GDAP1 variants in our study presented axonal type of neuropathy which was consist with previous studies in Chinese patients except one of patients reported by He J (He et al., 2018). Actually, this case showed an absent MNCV and CMAPs in median nerve as no response by electrophysiological detection that caused difficulty in defining its clinical type. And a study conducted in a cohort of 1,030 Japanese patients with inherited peripheral neuropathies showed that all 10 patients with GDAP1 pathogenic variants had axonal type peripheral neuropathy. However, patients presented demyelinating type were not uncommon in European and Mediterranean populations (Ammar et al., 2003; Baxter et al., 2002; Boerkoel et al., 2003; Georgiou et al., 2006; Parman et al., 2004).

To further characterize the pathogenicity of novel GDAP1 variants, functional studies were applied to verify the effect of the novel splicing variant $(\mathrm{c} .694+1 \mathrm{G}>\mathrm{A})$ on pre-mRNA and the biological effects of two novel variants (p.L26R and p.S169fs). It is well known that GDAP1, located in the external mitochondrial membrane, presents a ubiquitous distribution in various human tissues and mainly expressed in neurons, and is involved in many aspects of mitochondrial morphology and functioning (Niemann et al., 2005; Pedrola et al., 2008). In the present study, transmission electron microscopy showed a distinct disruption of the mitochondrial membrane and disorganization of cristae in the muscular tissue. These findings indicated that GDAP1 may play an important role in impair cellular energetics, and consequently, in cellular function (Benard et al., 2007). Fibroblasts from autosomal-recessive CMT2K patients showed reduced GDAP1 expression levels, fragmentation of the mitochondrial, a declining mitochondrial membrane potential, respiration defects and decreased cellular and mitochondrial ATP production. The p.S169fs led to the appearance of the premature stop codon. It could result in rapid degradation of GDAP1 protein and cause severe phenotype with disease onset in the patient's first decade (Cassereau et al., 2011; Niemann et al., 2005). Decreased MMP $(\Delta \psi \mathrm{m})$ and mitochondrial ATP production not only indicated cellular injury but also triggered the fragmentation of mitochondria (Legros, Lombes, Frachon, \& Rojo, 2002). In addition, the OCR assay showed respiratory deficiency in mutant fibroblasts could result in decreased efficiency of the mitochondrial ATP synthesis. To sum up, all these results showed that mitochondrial-mediated pathway plays a key role in the pathogenesis of $G D A P 1$ and can ultimately be characterized as the pathogenicity of these two variants.

In conclusion, we identified and classified the pathogenicity of three novel GDAP1 variants, which broaden the genetic spectrum of GDAP1. Further we described the clinical features of CMT patients carrying pathogenic variants in GDAP1 and found that almost all Chinese CMT patients with GDAP1 variants present axonal type. Additionally, we identified the ultrastructure damage of mitochondrial and emphasized the function of GDAP1 in mitochondrial-mediated pathway.

\section{Acknowledgements}

The authors sincerely thank the participants for their help and willingness to participate in this study. This work was supported by grants from the National Natural Science Foundation of China (81125009, 
31871022), the Fundamental Research Funds for the Central Universities (2019XZZX001-01-04) and the research foundation for distinguished scholar of Zhejiang University to Zhi-Ying Wu (188020-193810101/089).

\section{Conflict of Interest}

All authors declare no conflicts of interest.

\section{References}

Abe, A., Numakura, C., Kijima, K., Hayashi, M., Hashimoto, T., \& Hayasaka, K. (2011). Molecular diagnosis and clinical onset of Charcot-Marie-Tooth disease in Japan. $J$ Hum Genet, 56 (5), 364-368. doi:10.1038/jhg.2011.20

Ammar, N., Nelis, E., Merlini, L., Barisic, N., Amouri, R., Ceuterick, C., . . . De Jonghe, P. (2003). Identification of novel GDAP1 mutations causing autosomal recessive Charcot-Marie-Tooth disease.Neuromuscul Disord, 13 (9), 720-728. doi:10.1016/s0960-8966(03)00093-2

Azzedine, H., Ruberg, M., Ente, D., Gilardeau, C., Perie, S., Wechsler, B., . . . Dubourg, O. (2003). Variability of disease progression in a family with autosomal recessive CMT associated with a S194X and new R310Q mutation in the GDAP1 gene. Neuromuscul Disord, 13 (4), 341-346.

Baxter, R. V., Ben Othmane, K., Rochelle, J. M., Stajich, J. E., Hulette, C., Dew-Knight, S., . . . Vance, J. M. (2002). Ganglioside-induced differentiation-associated protein-1 is mutant in Charcot-Marie-Tooth disease type 4A/8q21. Nat Genet, 30 (1), 21-22. doi:10.1038/ng796

Ben Othmane, K., Hentati, F., Lennon, F., Ben Hamida, C., Blel, S., Roses, A. D., . . . Vance, J. M. (1993). Linkage of a locus (CMT4A) for autosomal recessive Charcot-Marie-Tooth disease to chromosome 8q. Hum Mol Genet, 2 (10), 1625-1628. doi:10.1093/hmg/ 2.10 .1625

Benard, G., Bellance, N., James, D., Parrone, P., Fernandez, H., Letellier, T., \& Rossignol, R. (2007). Mitochondrial bioenergetics and structural network organization. J Cell Sci, 120 (Pt 5), 838-848. doi: $10.1242 /$ jcs.03381

Boerkoel, C. F., Takashima, H., Nakagawa, M., Izumo, S., Armstrong, D., Butler, I., . . . Lupski, J. R. (2003). CMT4A: identification of a Hispanic GDAP1 founder mutation. Ann Neurol, 53 (3), 400-405. doi:10.1002/ana.10505

Cassereau, J., Chevrollier, A., Bonneau, D., Verny, C., Procaccio, V., Reynier, P., \& Ferre, M. (2011). A locus-specific database for mutations in GDAP1 allows analysis of genotype-phenotype correlations in Charcot-Marie-Tooth diseases type 4A and 2K. Orphanet J Rare Dis, 6 , 87. doi:10.1186/1750-1172-6-87

Chen, C. X., \& Dong, H. L., Wei, Q., Li, L. X., Yu, H., Li, J. Q., Liu, G. L., Li, H. F., Bai, G., Ma, H., \& Wu, Z. Y. (2019). Genetic spectrum and clinical profiles in a southeast Chinese cohort of Charcot-Marie-Tooth disease. Clin Genet, 96 (5), 439-448. doi:10.1111/cge.13616

Chung, K. W., Hyun, Y. S., Lee, H. J., Jung, H. K., Koo, H., Yoo, J. H., . . . Choi, B. O. (2011). Two recessive intermediate Charcot-Marie-Tooth patients with GDAP1 mutations. J Peripher Nerv Syst, 16 (2), 143-146. doi:10.1111/j.1529-8027.2011.00329.x

Claramunt, R., Pedrola, L., Sevilla, T., Lopez de Munain, A., Berciano, J., Cuesta, A., . . . Palau, F. (2005). Genetics of Charcot-Marie-Tooth disease type 4A: mutations, inheritance, phenotypic variability, and founder effect. J Med Genet, 42 (4), 358-365. doi:10.1136/jmg.2004.022178

Estela, A., Pla-Martin, D., Sanchez-Piris, M., Sesaki, H., \& Palau, F. (2011). Charcot-Marie-Tooth-related gene GDAP1 complements cell cycle delay at G2/M phase in Saccharomyces cerevisiae fis1 gene-defective cells. J Biol Chem, 286 (42), 36777-36786. doi:10.1074/jbc.M111.260042

Fernandez-Lizarbe, S., Civera-Tregon, A., Cantarero, L., Herrer, I., Juarez, P., Hoenicka, J., \& Palau, F. (2019). Neuroinflammation in the pathogenesis of axonal Charcot-Marie-Tooth disease caused by lack of 
GDAP1. Exp Neurol, 320 , 113004. doi:10.1016/j.expneurol. 2019.113004

Fu, J., Dai, S., Lu, Y., Wu, R., Wang, Z., Yuan, Y., \& Lv, H. (2017). Similar clinical, pathological, and genetic features in Chinese patients with autosomal recessive and dominant Charcot-Marie-Tooth disease type 2K. Neuromuscul Disord, 27 (8), 760-765. doi:10.1016/j.nmd. 2017.04.001

Georgiou, D. M., Nicolaou, P., Chitayat, D., Koutsou, P., Babul-Hirji, R., Vajsar, J., . . . Christodoulou, K. (2006). A novel GDAP1 mutation 439delA is associated with autosomal recessive CMT disease. Can $J$ Neurol Sci, 33 (3), 311-316. doi:10.1017/s03171671 00005199

He, J., Guo, L., Xu, G., Xu, L., Lin, S., Chen, W., \& Wang, N. (2018). Clinical and genetic investigation in Chinese patients with demyelinating Charcot-Marie-Tooth disease. J Peripher Nerv Syst, 23 (4), 216-226. doi:10.1111/jns.12277

Legros, F., Lombes, A., Frachon, P., \& Rojo, M. (2002). Mitochondrial fusion in human cells is efficient, requires the inner membrane potential, and is mediated by mitofusins. Mol Biol Cell, 13 (12), 4343-4354. doi:10.1091/mbc.e02-06-0330

Li, L. X., Liu, G. L., Liu, Z. J., Lu, C., \& Wu, Z. Y. (2017). Identification and functional characterization of two missense mutations in NDRG1 associated with Charcot-Marie-Tooth disease type 4D. Hum Mutat, 38 (11), 1569-1578. doi:10.1002/humu.23309

Lin, K. P., Soong, B. W., Yang, C. C., Huang, L. W., Chang, M. H., Lee, I. H., . . . Lee, Y. C. (2011). The mutational spectrum in a cohort of Charcot-Marie-Tooth disease type 2 among the Han Chinese in Taiwan.PLoS One, 6 (12), e29393. doi:10.1371/journal.pone.0029393

Lopez Del Amo, V., Seco-Cervera, M., Garcia-Gimenez, J. L., Whitworth, A. J., Pallardo, F. V., \& Galindo, M. I. (2015). Mitochondrial defects and neuromuscular degeneration caused by altered expression of Drosophila Gdap1: implications for the Charcot-Marie-Tooth neuropathy.Hum Mol Genet, 24 (1), 21-36. doi:10.1093/hmg/ddu416

Manganelli, F., Pisciotta, C., Nolano, M., Capponi, S., Geroldi, A., Topa, A., . . . Santoro, L. (2012). A novel autosomal dominant GDAP1 mutation in an Italian CMT2 family. J Peripher Nerv Syst, 17 (3), 351-355. doi:10.1111/j.1529-8027.2012.00414.x

Manganelli, F., Tozza, S., Pisciotta, C., Bellone, E., Iodice, R., Nolano, M., . . . Santoro, L. (2014). Charcot-Marie-Tooth disease: frequency of genetic subtypes in a Southern Italy population. $J$ Peripher Nerv Syst, 19 (4), 292-298. doi:10.1111/jns.12092

Niemann, A., Ruegg, M., La Padula, V., Schenone, A., \& Suter, U. (2005). Ganglioside-induced differentiation associated protein 1 is a regulator of the mitochondrial network: new implications for Charcot-MarieTooth disease. J Cell Biol, 170 (7), 1067-1078. doi:10.1083/ jcb.200507087

Niemann, A., Wagner, K. M., Ruegg, M., \& Suter, U. (2009). GDAP1 mutations differ in their effects on mitochondrial dynamics and apoptosis depending on the mode of inheritance. Neurobiol Dis, 36 (3), 509-520. doi:10.1016/j.nbd.2009.09.011

Pakhrin, P. S., Xie, Y., Hu, Z., Li, X., Liu, L., Huang, S., . . . Zhang, R. (2018). Genotype-phenotype correlation and frequency of distribution in a cohort of Chinese Charcot-Marie-Tooth patients associated with GDAP1 mutations. J Neurol, 265 (3), 637-646. doi:10.1007/ s00415-018-8743-9

Parman, Y., Battaloglu, E., Baris, I., Bilir, B., Poyraz, M., Bissar-Tadmouri, N., . . . Said, G. (2004). Clinicopathological and genetic study of early-onset demyelinating neuropathy. Brain, 127 (Pt 11), 25402550. doi:10.1093/brain/awh275

Pedrola, L., Espert, A., Valdes-Sanchez, T., Sanchez-Piris, M., Sirkowski, E. E., Scherer, S. S., . . . Palau, F. (2008). Cell expression of GDAP1 in the nervous system and pathogenesis of Charcot-Marie-Tooth type 4A disease. J Cell Mol Med, 12 (2), 679-689. doi:10.1111/ j.1582-4934.2007.00158.x 
Pezzini, I., Geroldi, A., Capponi, S., Gulli, R., Schenone, A., Grandis, M., . . . Bellone, E. (2016). GDAP1 mutations in Italian axonal Charcot-Marie-Tooth patients: Phenotypic features and clinical course.Neuromuscul Disord, 26 (1), 26-32. doi:10.1016/j.nmd.2015.09.008

Pla-Martin, D., Rueda, C. B., Estela, A., Sanchez-Piris, M., Gonzalez-Sanchez, P., Traba, J., . . . Palau, F. (2013). Silencing of the Charcot-Marie-Tooth disease-associated gene GDAP1 induces abnormal mitochondrial distribution and affects $\mathrm{Ca} 2+$ homeostasis by reducing store-operated Ca2+ entry. Neurobiol Dis, 55 , 140-151. doi:10.1016/j.nbd.2013.03.010

Sivera, R., Sevilla, T., Vilchez, J. J., Martinez-Rubio, D., Chumillas, M. J., Vazquez, J. F., . . . Espinos, C. (2013). Charcot-Marie-Tooth disease: genetic and clinical spectrum in a Spanish clinical series.Neurology, 81 (18), 1617-1625. doi:10.1212/WNL. 0b013e3182 a9f56a

Yoshimura, A., Yuan, J. H., Hashiguchi, A., Hiramatsu, Y., Ando, M., Higuchi, Y., . . . Takashima, H. (2017). Clinical and mutational spectrum of Japanese patients with Charcot-Marie-Tooth disease caused by GDAP1 variants. Clin Genet, 92 (3), 274-280. doi:10.1111/cge.13002

Zhang, R. X., Tang, B. S., Zi, X. H., Luo, W., Xia, K., Pan, Q., . . . Li, X. B. (2004). [Mutation analysis of ganglioside-induced differentiation associated protein-1 gene in Chinese Charcot-Marie-Tooth disease]. Zhonghua Yi Xue Yi Chuan Xue Za Zhi, 21 (3), 207-210.

Zimon, M., Battaloglu, E., Parman, Y., Erdem, S., Baets, J., De Vriendt, E., . . . Jordanova, A. (2015). Unraveling the genetic landscape of autosomal recessive Charcot-Marie-Tooth neuropathies using a homozygosity mapping approach. Neurogenetics, 16 (1), 33-42. doi:10.1007/s10048-014-0422-0

\section{Legends of Figures}

Figure 1. GDAP1 variants identified in patients with CMT2K. (A) Pedigrees of three CMT families carried GDAP1 variants. Arrows indicate the proband of each family.(B) Sequence chromatograms of four variants in GDAP1 are shown. The lower chromatogram in each frame represents the variant, while the upper one represents the normal sequence. (C) Domain structure of GDAP1 protein and the location of variants in the GDAP1 protein in Chinese patients. GDAP1 contains two typical glutathione-S transferases (GST-N and GST-C). A single transmembrane domain (TMD) at the extremity of the C-terminal. A hydrophobic domain (HD) in the flanking $\mathrm{C}$ terminal region and two additional regions constituting the $\alpha 4-\alpha 5$ loop. Variants identified in our team are in red and variants reported in other Chinese study are in black.

Figure 2. Splicing alteration was identified by a minigene assay. (A) cDNA products were separated by agarose gel electrophoresis. Lane1: Marker; Lane2: WT [263 bp + 115bp (exon 5)]; Lane3: c.694+1G>A variant $263 \mathrm{bp}$. (B) The sequencing results for the bands. (C) Schematic diagram of minigene construction. Splice donor (SD) and splice acceptor (SA) are two exons of the pSPL3 vector.

Figure 3. Transmission electron microscopic analysis of mitochondrial structure in muscle tissue. (A) The mitochondrial structure of the control subject. (B-D) Compared with the control, GDAP1 mutations resulted in damage to mitochondria, including disruption of the mitochondrial membrane and disorganization of cristae (red arrows).

Figure 4. Function studies of mutant GDAP1 and control in muscle tissue and patient-derived fibroblasts. (A) Western blot analysis of protein extracts obtained from muscle tissue of proband 1. The anti-GDAP1 antibody was used to detect the protein. (B)Values represent mean $\pm \mathrm{SD}(\mathrm{n}=3$, ****P $<0.0001$ ).(C) Staining of mitochondria marker (Mitotraker, red) and DAPI (blue) in fibroblasts from the Control (upper panel) and the patient (lower panel). Insets in the images are enlarged (original magnification, $\times 4.0)$ to the left bottom. Scale bar, $20 \mu \mathrm{m}$. (D)TMRM staining was used to measure MMP $(\Delta \psi \mathrm{m})$ in fibroblasts from the Control (upper panel) and the patient (bottom right panel). FCCP was used as the positive control. Scale bar, $200 \mu \mathrm{m}$. (E)Statistical analysis of $\mathrm{D}\left(\mathrm{n}=3\right.$, $\left.{ }^{* *} \mathrm{P}<0.01\right)$. (F)Measurement of cellular ATP levels using bioluminescence assay. Fibroblast were incubated with $10 \mathrm{mM}$ glucose and ATP bioluminometers analysis revealed a significant decrease of ATP content in the patient-derived fibroblast 
$\left(\mathrm{n}=5,{ }^{*} * * * \mathrm{P}<0.0001\right)$. (G)Measurement of mitochondrial ATP levels using bioluminescence assay. Fibroblast were incubated with $5 \mathrm{mM}$ 2-deoxy-D-glucose plus $5 \mathrm{mM}$ pyruvate which can inhibit the glycolysis. The result revealed a significant decrease of ATP content in the patient-derived fibroblast $(\mathrm{n}=5, * * * * \mathrm{P}<0.0001)$. (H) An analysis of $\mathrm{O} 2$ consumption in the mutant and control fibroblast using different inhibitors. The rates of $\mathrm{O} 2(\mathrm{OCR})$ were first measured on $2 \times 10^{4}$ cells under basal condition and then sequentially added to oligomycin $(1.5 \mu \mathrm{M})$, carbonyl cyanide p-(trifluoromethoxy) phenylhydrazone (FCCP) $(0.5 \mu \mathrm{M})$, rotenone $(1 \mu \mathrm{M})$ and antimycin $\mathrm{A}(1 \mu \mathrm{M})$ at indicated times to determine different parameters of mitochondrial functions. (I) Graphs presented the ATP-linked OCR, proton leak OCR, maximal OCR, reserve capacity and non-mitochondrial OCR in mutant and control fibroblast. Non-mitochondrial OCR was determined as the OCR after rotenone/antimycin A treatment. Basal OCR was determined as OCR before oligomycin minus OCR after rotenone/antimycin A. ATP-linked OCR was determined as OCR before oligomycin minus OCR after oligomycin. Proton leak was determined as basal OCR minus ATP-linked OCR. Maximal was determined as the OCR after FCCP minus non-mitochondrial OCR. Reserve capacity was defined as the difference between maximal OCR after FCCP minus basal OCR. The average of four determinations for each fibroblast is shown.

A

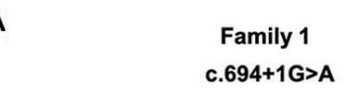

I

II

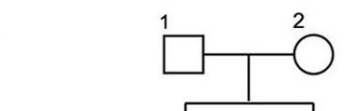

III

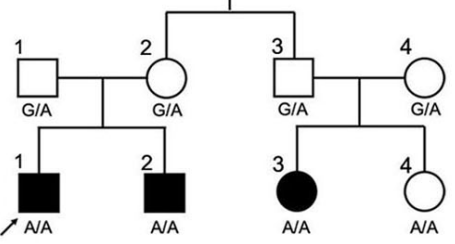

Family 2

p.L26R/p.S169fs

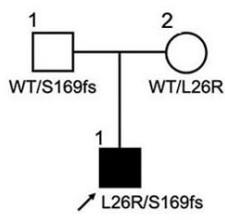

Family 3

p.R120W

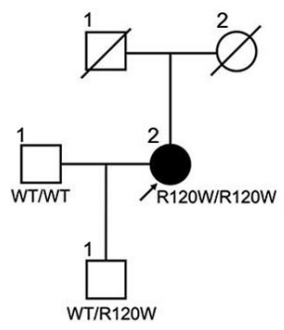

B

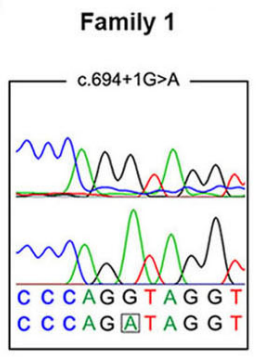

Family 2

Family 3
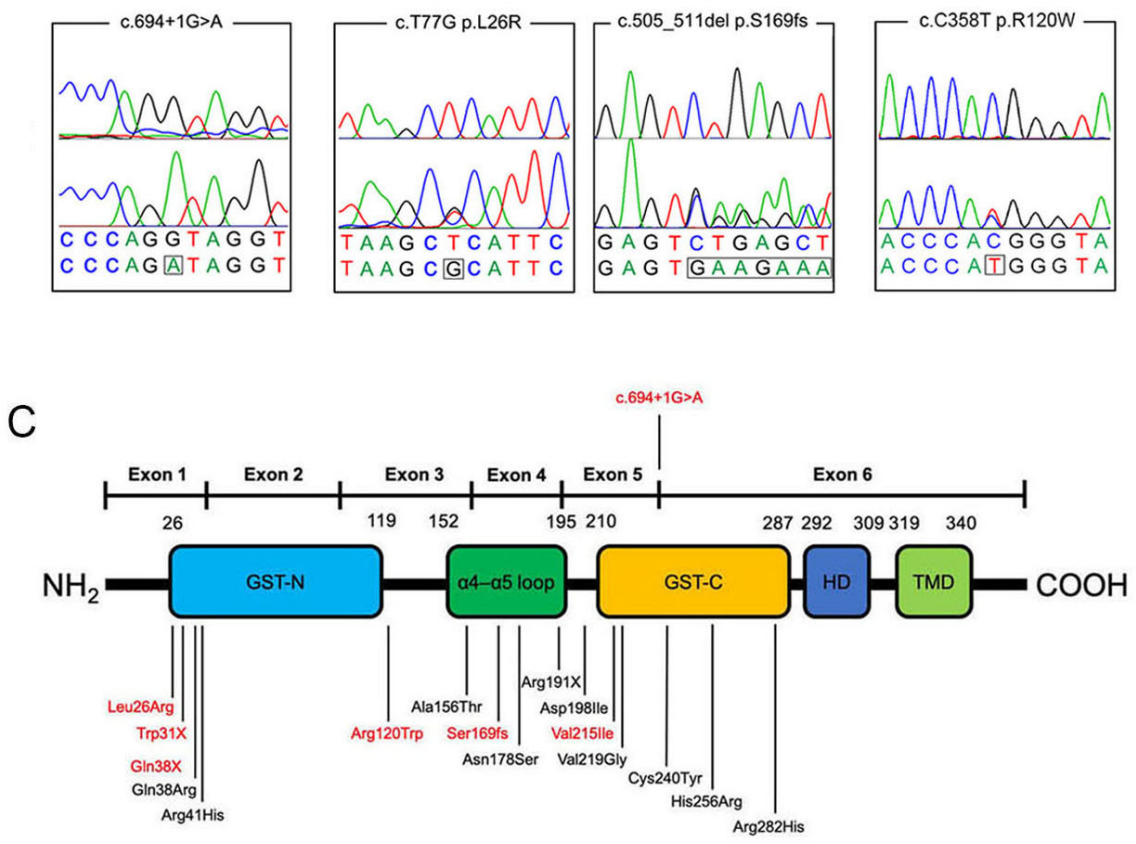
A

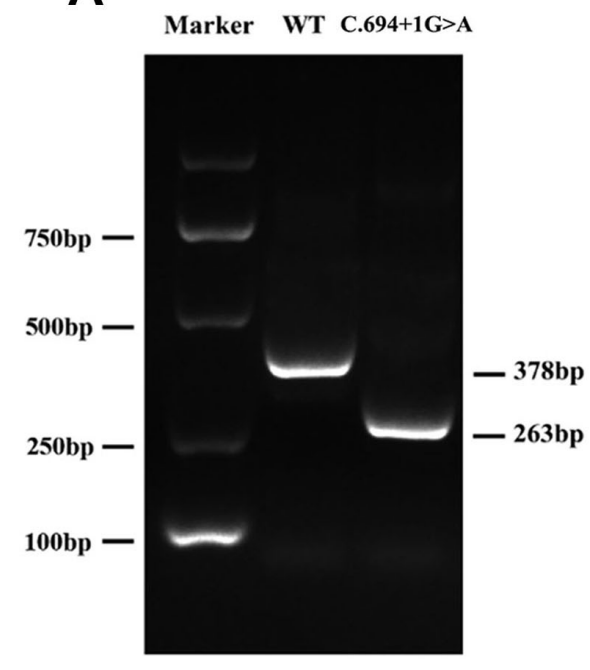

B

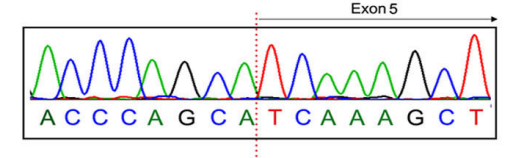

Wh M M

C
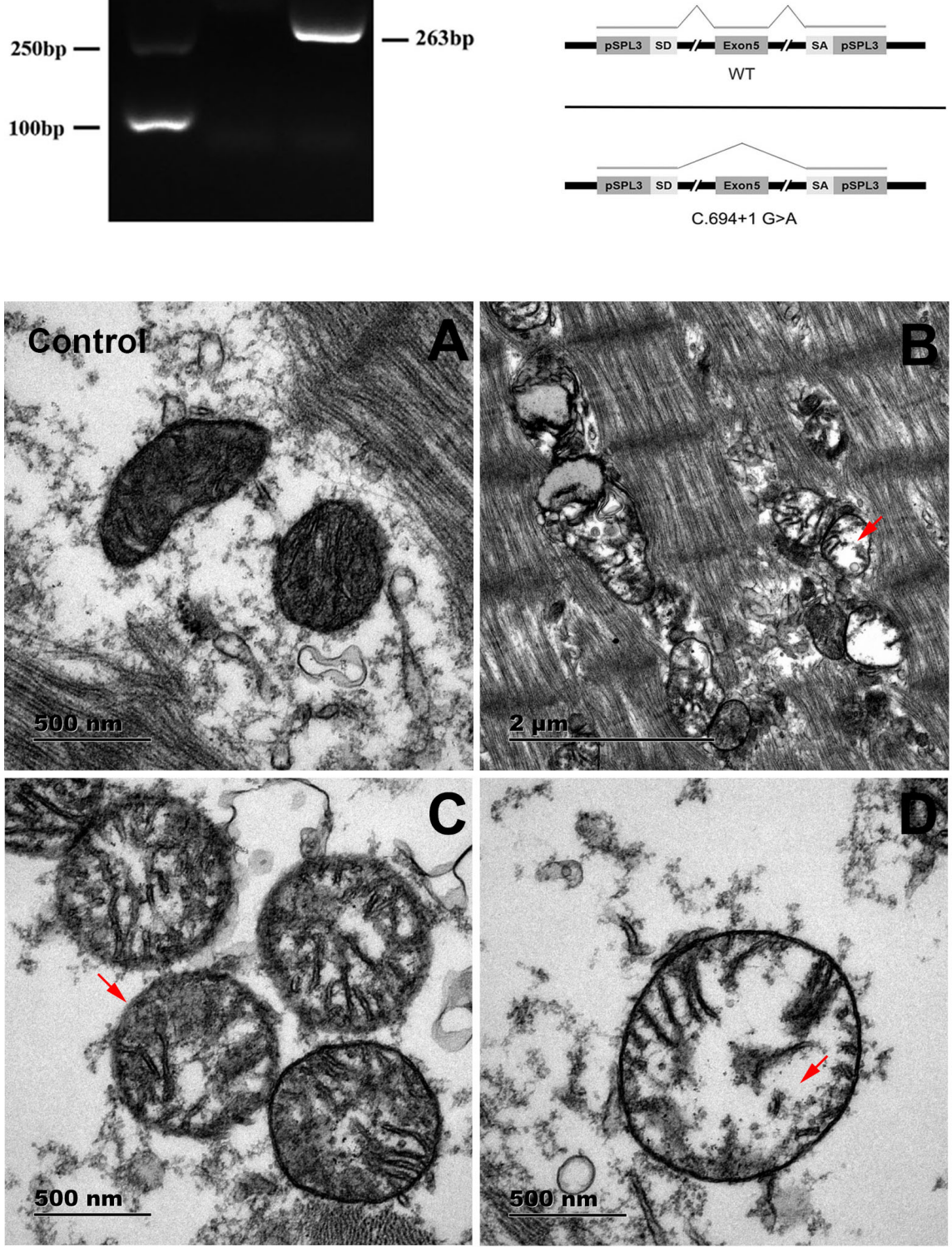
A

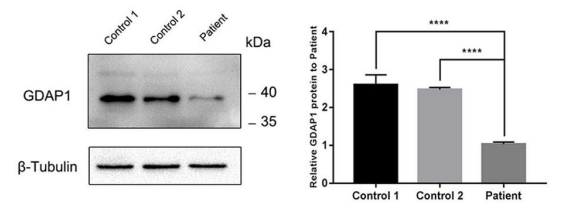

D

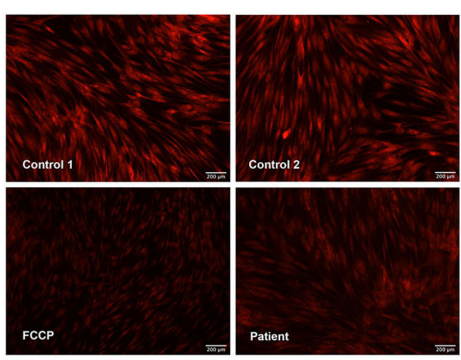

F

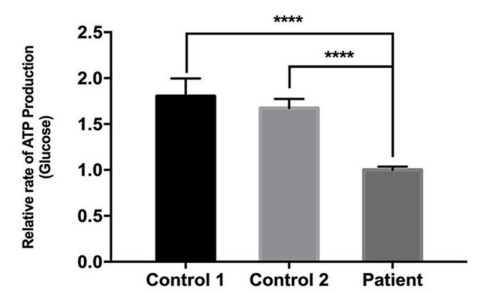

G

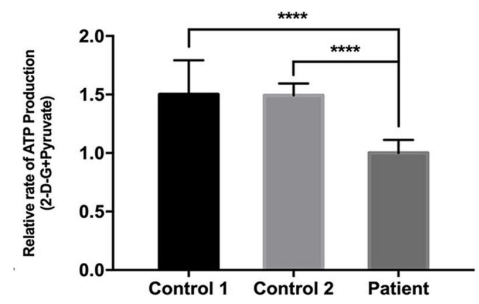

C

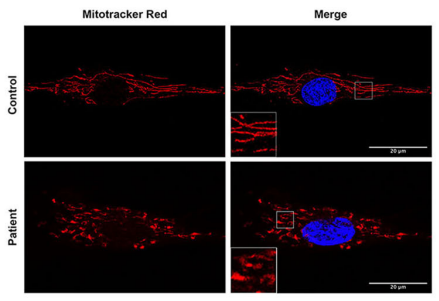

E

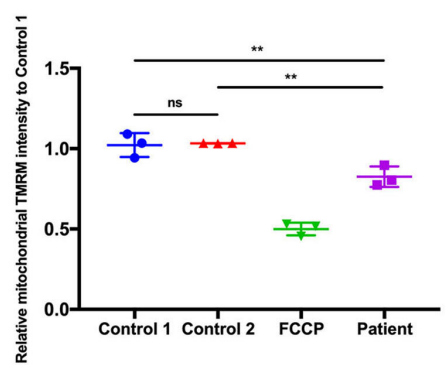

$\mathrm{H}$

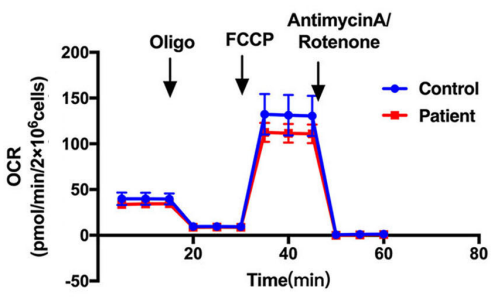

I

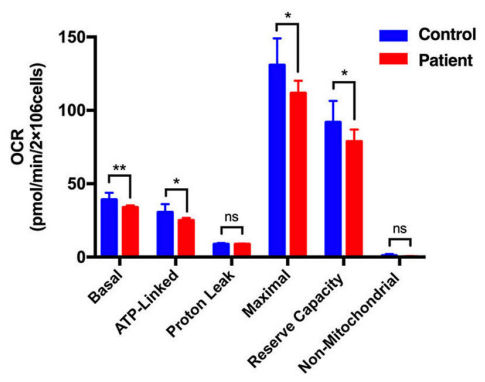

\section{Hosted file}

Table 1.docx available at https://authorea.com/users/328789/articles/455945-identificationand-functional-characterization-of-novel-gdap1-variants-in-chinese-patients-withcharcot-marie-tooth-disease

\section{Hosted file}

Table 2.docx available at https://authorea.com/users/328789/articles/455945-identificationand-functional-characterization-of-novel-gdap1-variants-in-chinese-patients-withcharcot-marie-tooth-disease 


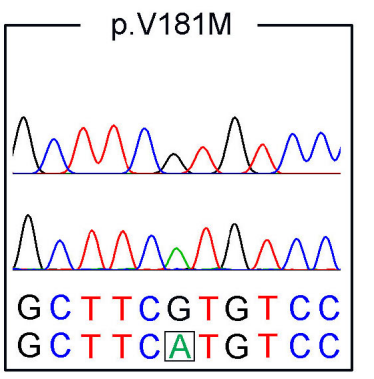

GJB1

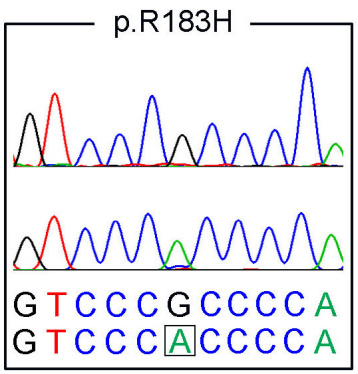

GJB1
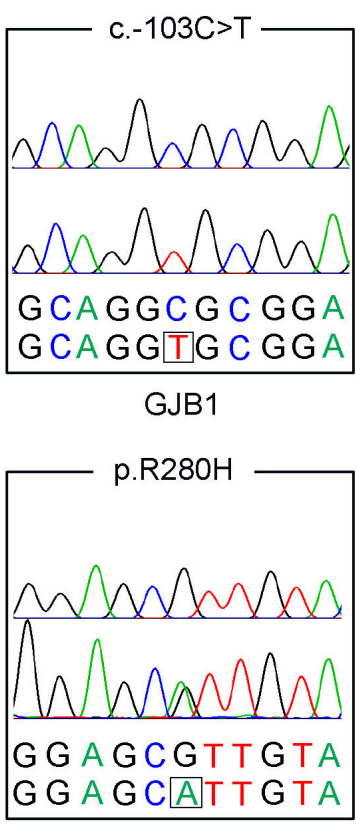

MFN2
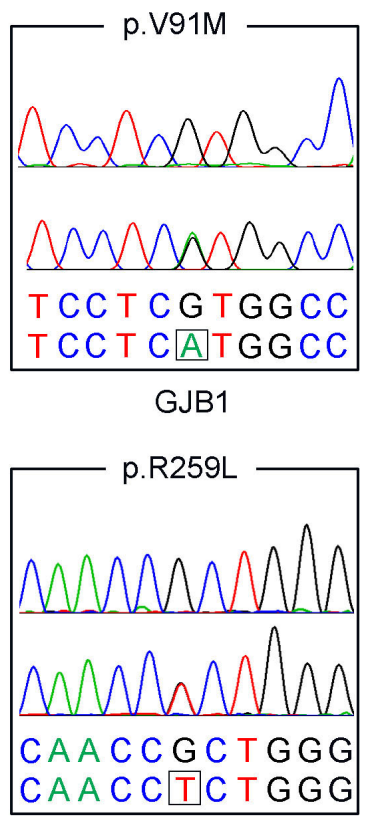

MFN2
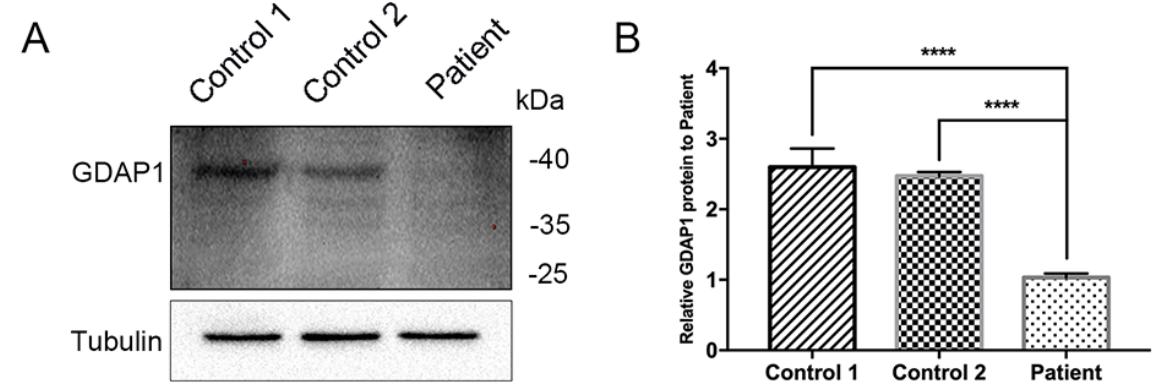

C
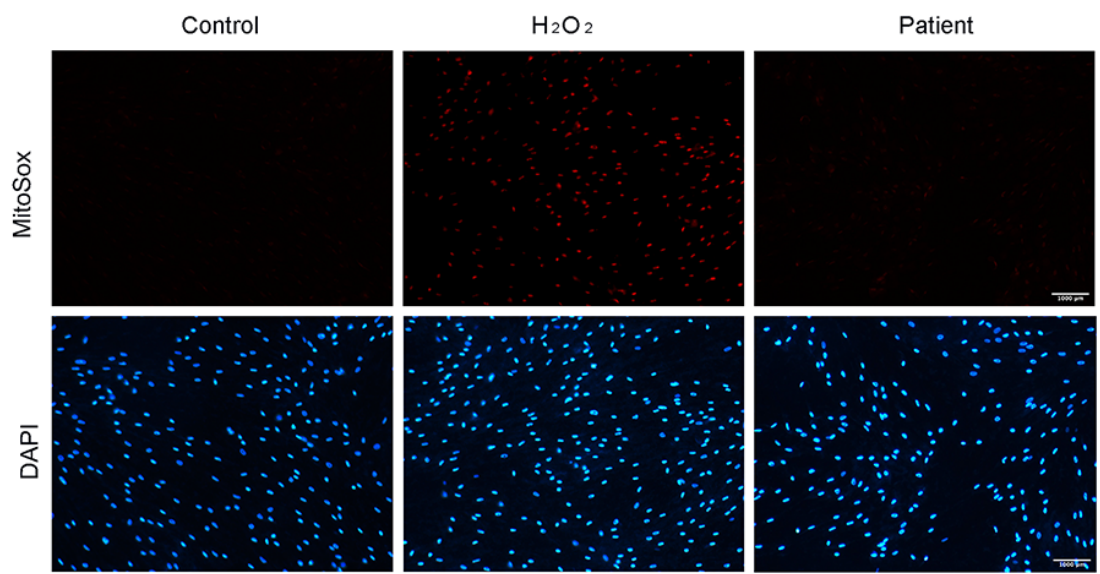